\title{
A Two-Terminal IC Temperature Transducer
}

\author{
MICHAEL P. TIMKO, MEMBER, IEEE
}

\begin{abstract}
A monolithic IC temperature transducer with an operating temperature range of $-125^{\circ} \mathrm{C}$ to $+200^{\circ} \mathrm{C}$ has been designed, fabricated, and tested. The two-terminal device, which is fabricated using laser trimmed thin-film-on-silicon technology, is a calibrated temperaturedependent current source with an average output impedence of $10 \mathrm{M} \Omega$ over the $3.5-\mathrm{V}$ to $30-\mathrm{V}$ range of input voltage. Overall absolute accuracies of $\pm 0.5^{\circ} \mathrm{C}$ from $-75^{\circ} \mathrm{C}$ to $+150^{\circ} \mathrm{C}$ have been achieved on a scale of $1 \mu \mathrm{A} / \mathrm{K}$ under optimum operating conditions.
\end{abstract}

\section{INTRODUCTION}

$\mathrm{S}$ ILICON bipolar transistors have a property which can be exploited to produce a voltage that is directly proportional to absolute temperature (PTAT). If the transistors are designed so as to minimize other temperature effects, this voltage will be a highly accurate representation of the temperature over a wide range. A temperature sensor, however, must have several other important properties to be truly flexible and widely useful. A list of specifications for all temperature transducers includes the following:

1) accuracy versus temperature (the predictability of the output parameter);

2) linearity (the constancy of the change in output parameter per degree over the temperature range);

3) interchangeability (absolute calibration);

4) signal level;

5) remote sensing capability (the maximum allowable distance between the sensor and supporting electronics and the number and quality of connections necessary);

6) temperature range;

7) cost [including support electronics to allow for deficiencies in 2)-5)].

While an IC temperature transducer probably cannot be as accurate as, for instance, a platinum resistance thermometer and the temperature range is limited to about $-125^{\circ} \mathrm{C}$ to $+200^{\circ} \mathrm{C}$, the flexibility of monolithic integration allows the designer to include many features that no other medium can achieve.

Previous IC designs using the PTAT voltage specifically to sense temperature concentrated on amplifying and buffering the voltage over a limited temperature range with only rough calibration [1]. In contrast, the emphasis in this new design is the best achievable accuracy over the widest possible temperature range. This goal has reached a point never before obtained in an integrated circuit by combining a unique circuit configuration with the latest laser-trimmed thin-film resistor technology to produce a calibrated PTAT current. The fact that the output parameter is current, and that the sensor

Manuscript received May 29, 1976; revised August 4, 1976.

The author is with the Semiconductor Division, Analog Devices, Wilmington, MA 01887. is relatively insensitive to the voltage across it, makes the device easy to use, even over long wires.

\section{Temperature Dependence}

The temperature sensitive parameter of interest is the difference between the $V_{b e}$ 's of two transistors operating at a constant ratio of collector current densities. This can be derived from the equation for emitter current density [2] :

$$
J_{e}=\frac{1}{\alpha} J_{s}\left(e^{q V_{b e}} / k T-1\right)
$$

where

$J_{e} \quad$ emitter current density;

$J_{s} \quad$ emitter saturation current density;

$\alpha \quad$ common base current gain;

$V_{b e}$ base emitter voltage;

$q$ charge on an electron;

$k$ Boltzmann's constant:

and

\section{$T$ absolute temperature.}

If we assume $J_{e} \gg J_{s}$, then for two transistors at current densities $J_{e 1}$ and $J_{e 2}$ :

$$
V_{T}=V_{b e 1}-V_{b e 2}=\frac{k T}{q} \ln \frac{\alpha_{1} J_{e 1} J_{s 2}}{\alpha_{2} J_{e 2} J_{s 1}} .
$$

For $V_{T}$ to be proportional to absolute temperature, the logarithmic term must be constant. For well-matched transistors $\alpha_{1}=\alpha_{2}$ and $J_{s 1}=J_{s 2}$. (In the circuit, as long as these parameters remain in constant ratio over temperature, the resulting error will be removed by the calibration trim.)

$$
V_{T}=T \frac{k}{q} \ln r
$$$$
\text { If } J_{e 1} / J_{e 2} \text { is a constant } r \text {, then }
$$

There are several ways to achieve the desired ratio of current densities. Two identical transistors operated at two different collector currents, held at a constant ratio, could be used. However, a more practical method is to operate two transistors of unequal emitter areas at equal collector currents. In order to have control over this ratio, the larger transistor emitter can be made up of a number of parallel emitters similar to that of the smaller transistor. The area ratio is then accurately equal to the ratio of the number of emitters. (In the schematic diagrams in this paper, a number immediately adjacent to an emitter refers to the number of similar emitters in that transistor relative to other transistors of the same type, n-p-n or $p-n-p$.)

Fig. 1 is an elementary application of the above principle. Neglecting for the moment the variation of $I_{c}$ with $V_{c e}$ and the 


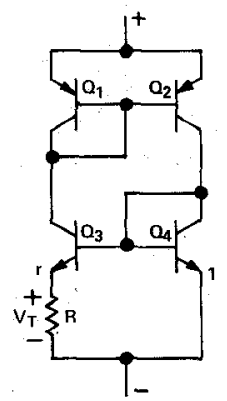

Fig. 1. Elementary example of a PTAT generating circuit.

effects of base current, this circuit will conduct a current proportional to absolute temperature for any voltage greater than two $V_{b e}$ across the device. Transistors $Q_{1}$ and $Q_{2}$ constrain the collector currents of $Q_{3}$ and $Q_{4}$ to be equal. Since the emitter area ratio of $Q_{3}$ and $Q_{4}$ is $r$, the difference in their $V_{b e}$ 's is $V_{T}$ in (3). For $r=8$,

$$
V_{T}=\frac{k T}{q} \ln 8=T \times 0.1792 \mathrm{mV} / \mathrm{K}
$$

or $53.44 \mathrm{mV}$ at $25^{\circ} \mathrm{C}$.

This voltage, when amplified and buffered, would make a useful output parameter. But, since $V_{T}$ is impressed across resistor $r$, the current drawn by each side of the circuit is

$$
I=\frac{V_{T}}{R}=\frac{k T}{q} \frac{\ln r}{R} .
$$

If $R$ has a temperature coefficient of zero, then the total current drawn by the circuit is also proportional to absolute temperature. Under the original assumptions for this elementary circuit, the supply current would be independent of input voltage (above two $V_{b e}$ 's) and the output impedence would be infinite.

Naturally, the circuit of Fig. 1 is insufficient for good results when made with real components. The Early effect and finite $\beta$ would reduce the output impedance drastically, the circuit would not start up dependably, and once started, it would probably oscillate under some otherwise useful conditions.

\section{The New Circuit: DC Accuracy}

Fig. 2 is a simplified schematic of the new circuit in which the idealized principle of Fig. 1 can be approached with real components. Transistors $Q_{1}, Q_{3}, Q_{9}$, and $Q_{11}$ correspond to the four transistors of Fig. 1. These transistors make up what will be referred to as the PTAT core since they define the temperature dependence of the circuit.

The collector currents of $Q_{1}$ and $Q_{3}$ are equal assuming only equal emitter areas and equal voltages at the bases of $Q_{7}$ and $Q_{8}$. The collector currents of $Q_{9}$ and $Q_{11}$ are forced to be equal to each other and to those of $Q_{1}$ and $Q_{3}$ by the com. bined action of $Q_{7}$ and $Q_{8}$.

Bias current for $Q_{7}$ and $Q_{8}$ is provided by diode $Q_{10}$ such that no nonPTAT component is added to the total supply current. The terminal current of this configuration is then three times the current in each branch or

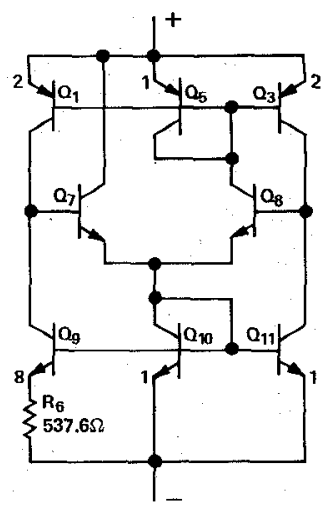

Fig. 2. Simplified schematic of the new circuit showing the major components contributing to the dc operation.

$$
I=3 \frac{k T}{q} \frac{\ln 8}{R_{6}} .
$$

Note that the value for $R_{6}$ on the schematic results in a scale factor of $1 \mu \mathrm{A} / \mathrm{K}$.

Transistor $Q_{5}$, which has half the emitter area of $Q_{1}$ or $Q_{3}$, operates at about half the current available from $Q_{10}$. As a result, the collector currents of $Q_{7}$ and $Q_{8}$ are nearly equal. The Early effect still causes errors between the two diodes and their associated transistors, creating an offset between $Q_{7}$ and $Q_{8}$. Since these errors are reduced from those of Fig. 1 by $n-p-n \beta$ (typically 400 ), they are insignificant except at temperature extremes.

Diode $Q_{10}$ is the key to the success of this circuit configuration. Its importance lies in the fact that it forces all currents to $V$ - that are not in the $Q_{1}, Q_{3}, Q_{9}, Q_{11}$ loop to sum to a PTAT current. Later, it is shown that even the hightemperature leakage currents can be measured in this way to extend the upper limit of operation.

In order to have good accuracy over temperature, $R_{6}$ must have a low temperature coefficient (TC). To put it another way, any TC in $R_{6}$ will show up as a TC in the absolute error of the sensor. This fact precludes the use of diffused resistors which have TC's of $\simeq 2000 \mathrm{ppm}$. SiCr thin-film resistors, however, can be made with consistent TC's of -30 to -50 $\mathrm{ppm} /{ }^{\circ} \mathrm{C}$. The effects of the aluminum interconnect and emitter resistance TC's are of the same order and were experimentally adjusted for optimum results. The inclusion of thin film also allows these devices to be laser trimmed at the wafer level to obtain excellent absolute accuracy as well as PTAT per. formance. Exact calibration allows for direct interchangeability of the finished devices.

\section{The Complete Circuit}

Fig. 3 is a complete schematic of the new circuit showing the additional components necessary to make the chip more closely approximate the ideal.

The absolute calibration of this device is accomplished by laser trimming $R_{5}$ and $R_{6}$ at the wafer level. Resistor $R_{5}$ was added to allow the initial current of the device to be either increased or decreased as needed so that the nominal design value could be the desired target value. The average amount 


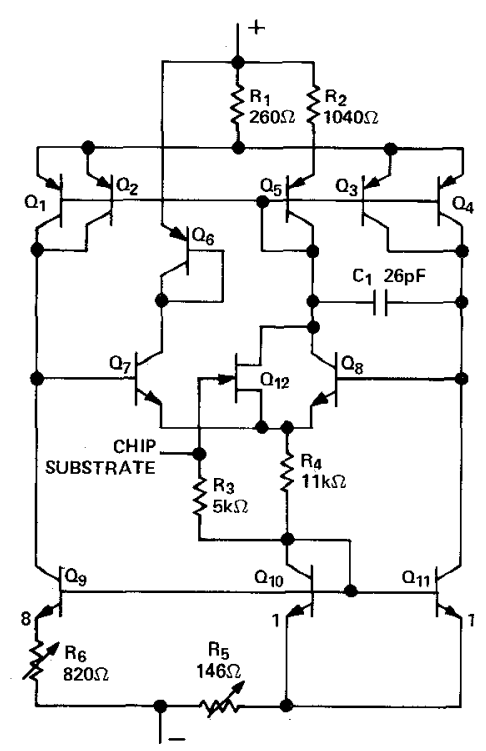

Fig. 3. Complete schematic of the two-terminal temperature transducer.

of trimming needed is thereby minimized and the yield is improved. The effective resistance for determining output current from $V_{T}$ is now $R_{6}-2 R_{5}$ since the emitter currents of $Q_{10}$ and $Q_{11}$ through $R_{5}$ cancel part of the voltage drop across $R_{6}$.

$Q_{12}$ provides a small current to start the device when power is applied. Note that this current finds its way to $V$-through $Q_{10}$ without contributing to output error.

As shown in the schematic, the gate of $Q_{12}$ (the chip substrate) is not connected to $V$ - as is normally the case in monolithic IC's. This unusual configuration is important in helping to extend the temperature range of the device well beyond the point at which leakage currents become significant. The single largest source of junction leakage current is the pocket containing $C_{1}$ and transistors $Q_{1}$ through $Q_{5}$. This current does not upset the balance of the PTAT core but, if simply brought out to $V$-, would add an error to the result. By connecting this and all other substrate currents to the positive side of $Q_{10}$, these currents are measured by the circuit. The leakage currents eventually accounted for the high temperature limit of the circuit by upsetting the balance of $Q_{7}$ and $Q_{8}$. At some point (about $210^{\circ} \mathrm{C}$ ) the collector current of $Q_{8}$ is reduced to zero and the device no longer functions. Instead of a direct connection between the substrate and $Q_{10}, R_{3}$ has been inserted to isolate certain substrate capacitances which otherwise would have a detrimental effect on the frequency stability of the circuit.

Since the substrate of this chip is not electrically connected to either output lead, special packaging considerations are required. The prototype devices were assembled in TO-52 metal transistor packages with an aluminum oxide substrate between the chip and the header. A special temperature sensor package would be necessary to realize the full capabilities of this device.

Resistor $R_{1}$ and $R_{2}$ were added to provide emitter degeneration and therefore reduce the base width modulation effects between $Q_{5}$ and the quad of lateral p-n-p's.
The combination of $R_{4}$ and $C_{1}$ determines the frequency compensation for the main feedback loop. The values shown, which were those used in the prototypes, somewhat overcompensate the circuit.

Transistor $Q_{6}$ balances the collector voltages of $Q_{7}$ and $Q_{8}$. More importantly, it provides protection for the device if the applied voltage is inadvertently reversed.

The simplicity of this design is a major factor in extending its useful temperature range. Additional components could be added to refine the accuracy at the cost of more parasitic leakage and a reduced upper temperature limit. The overall accuracy of the transducer compares very favorably with conventional sensors which are more difficult and less convenient to use.

\section{LAYOUT}

In this device, the layout design is as important as the circuit design. Fig. 4 is a chip photograph of a prototype device. While on-chip dissipation is not a major problem, the critical temperature sensing transistors $Q_{9}, Q_{10}$, and $Q_{11}$ are located at the opposite end of the chip from the main power consuming components $Q_{1}, Q_{2}, Q_{3}, Q_{4}, Q_{7}$, and $Q_{8}$. Note that $Q_{9}$ has eight large rectangular emitters with interdigitated base stripes. $Q_{11}$ is made in the same way with one identical emitter. (The two unconnected emitters were included for experimentation with other emitter ratios and current densities.) Note that the base box and isolation pocket of $Q_{11}$ are identical to those of $Q_{9}$. This provides first-order cancellation of leakage currents through these junctions at high temperatures.

$Q_{10}$ (which also has an extra unconnected emitter) has an additional interesting design feature. In an ordinary diodeconnected transistor, the combination of falling $V_{b e}$ and rising collector resistance produces saturation problems at high temperatures. $Q_{10}$ is designed to avoid this as shown in Fig. 5. The majority of the collector resistance of a monolithic $n-p-n$ transistor is in the epi between the buried layer and the 


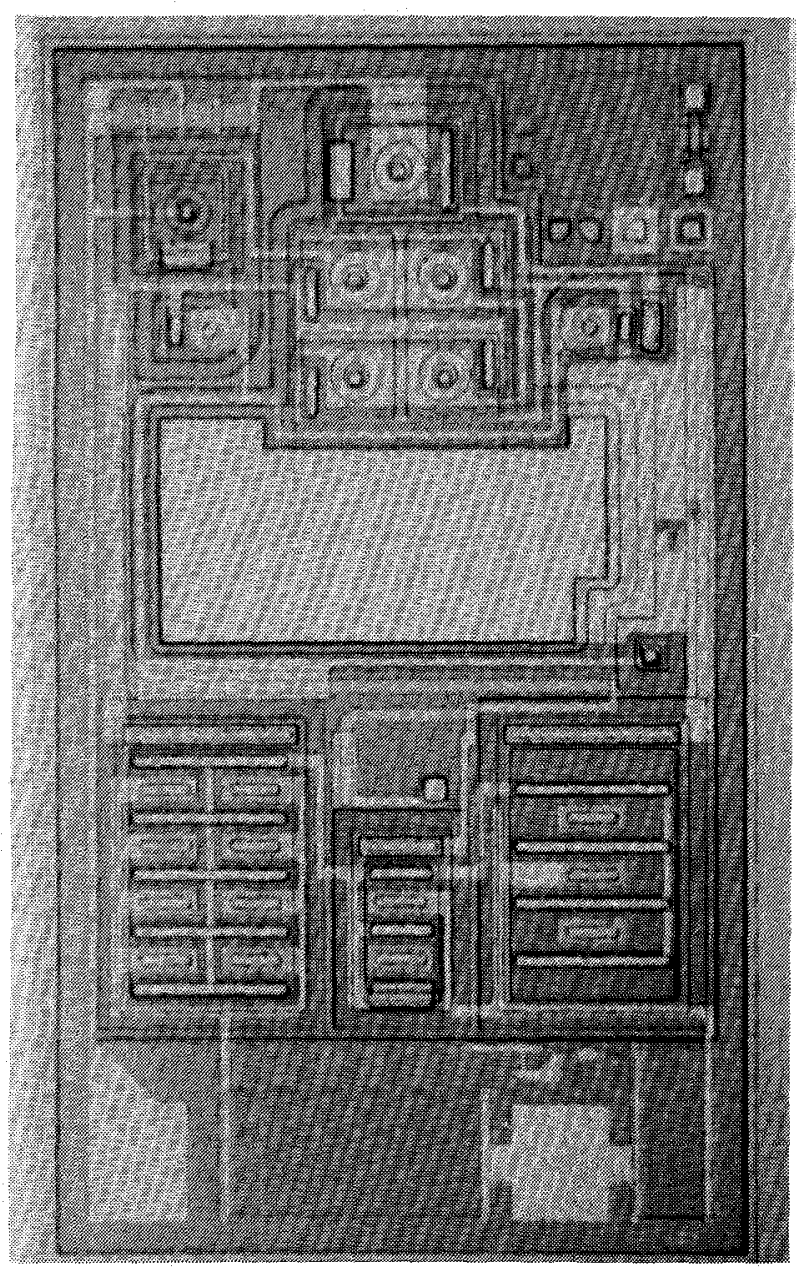

(a)

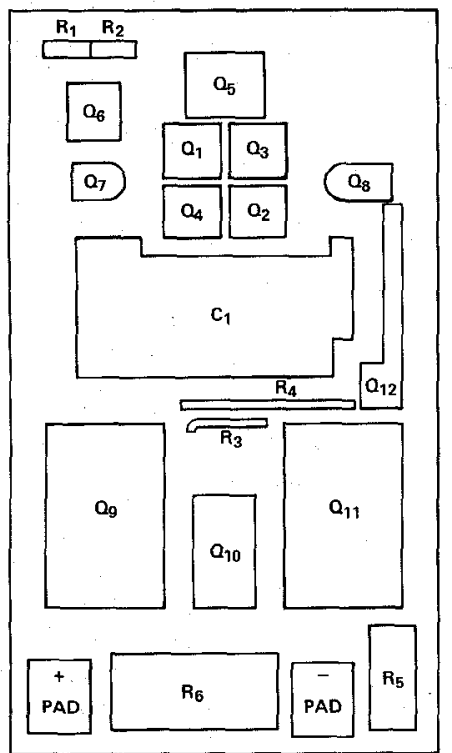

(b)

Fig. 4 (a) Photograph of the $37 \times 62$-mil chip. (b) Key to Fig. 4(a) showing the location of all components.

surface collector contact. By making two surface contacts on opposite ends of the transistor and using them as a Kelvin

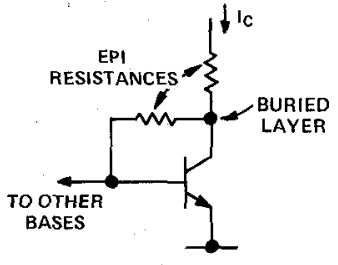

Fig. 5. Detail of diode $Q_{10}$.

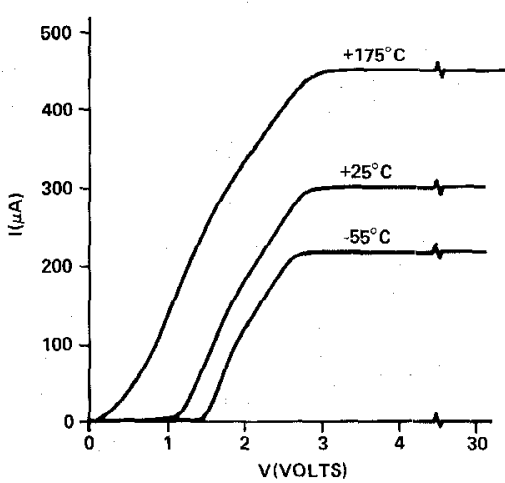

Fig. 6. V-I plot of a typical device at three temperatures.

contact, the voltage of buried layer is maintained near the base voltage over the whole temperature range. The voltage at the terminal into which the collector current flows changes with epi resistance, but this voltage is not critical to the accuracy of the circuit. While not as critical, $Q_{5}$ is also Kelvinconnected for the same reason.

Transistors $Q_{1}$ through $Q_{4}$ are wired as a cross-connected quad [3] and carefully matched in geometry and parasitics to insure faithful tracking over temperature.

\section{PERFORMANCE}

Fig. 6 shows the $V-I$ characteristic for voltage from zero through the operating region. While this particular device enters its linear region at about $2.7 \mathrm{~V}$, the minimum guarantee. able operating voltage, taking into account process variables, is $3.5 \mathrm{~V}$. The output impedance varies over the active region from $5 \mathrm{M} \Omega$ at $5 \mathrm{~V}$ to over $20 \mathrm{M} \Omega$ at $15 \mathrm{~V}$ and above. Actually, the output current changes no more than about 2-3 $\mu \mathrm{A}$ over the whole useful voltage range. Maintaining the voltage across the device within a volt of any operating point keeps this error below $0.2^{\circ} \mathrm{C}$.

A supply voltage of $5 \mathrm{~V}$ was chosen as a convenient level at which to trim the devices. All measurements for absolute accuracy were also made with $5 \mathrm{~V}$ across the device.

As mentioned earlier, these devices are adjusted to a scale factor of $1 \mu \mathrm{A} / \mathrm{K}$ by the laser wafer trim process discussed elsewhere [4]. The temperature of the wafer is carefully measured during the trim procedure and each device is then trimmed to indicate that temperature. This procedure presently yields a spread of results after assembly and burn-in such that about half the devices are accurate to within $\pm 1^{\circ} \mathrm{C}$ at $25^{\circ} \mathrm{C}$.

Fig. 7 shows the absolute error of three different devices between $-125^{\circ} \mathrm{C}$ and $+200^{\circ} \mathrm{C}$. These devices were selected for good calibration at $25^{\circ} \mathrm{C}$ in order to emphasize the relative 


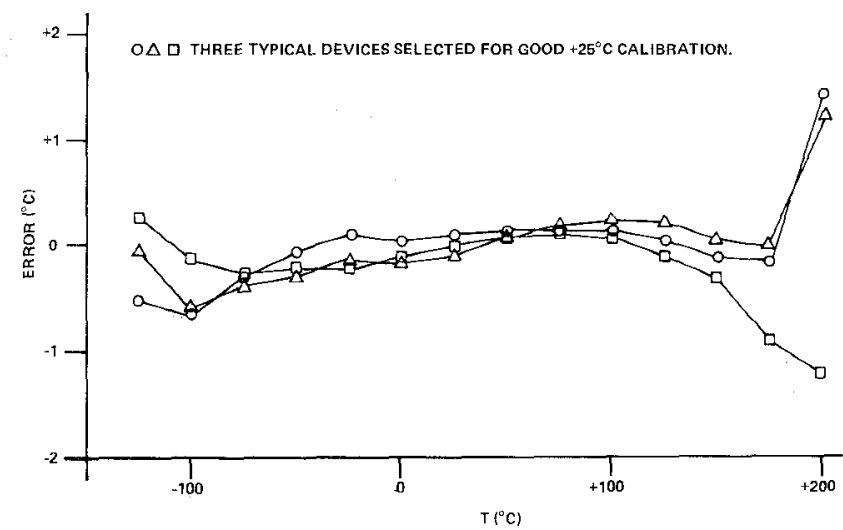

Fig. 7. Absolute error versus temperature.

linearity. The measurements were done by mounting the devices under test in an aluminum block suspended in a temperature chamber into which a calibrated thermocouple was also inserted. A direct comparison was made between the thermocouple and the devices when the block had settled near the desired temperature. The figure shows accuracy within $\pm 0.5^{\circ} \mathrm{C}$ from $-75^{\circ} \mathrm{C}$ to $+150^{\circ} \mathrm{C}$ on all three units. This nonlinearity over this range compares favorably with the only other IC temperature transducer available which specifies a nonlinearity of $1.8^{\circ} \mathrm{C}$ over the $-55^{\circ} \mathrm{C}$ to $+125^{\circ} \mathrm{C}$ range. Thermocouples, the most widely used sensors, have a standard limit of error of $\pm 0.8^{\circ} \mathrm{C}$ over a similar temperature range.

At the extremes of temperature, the inherent linearity is degraded but some devices remain useful for another $50^{\circ} \mathrm{C}$ on each end. At high temperatures, junction leakages, saturation resistances, and high-level injection effects eventually come into play. Most devices are useful at $+175^{\circ} \mathrm{C}$ and a selected few will operate at $+200^{\circ} \mathrm{C}$. At the low end, $\beta$ degradation and increasing current in $Q_{12}$ cause the errors. We found it very difficult to evaluate the devices below $-125^{\circ} \mathrm{C}$ because the test fixture would not settle long enough to do the measurements. The only other point we could get was to immerse the device and the thermocouple into liquid nitrogen. Most of the devices still functioned but the output impedance was seriously degraded and the accuracy at $5 \mathrm{~V}$ was no better than $\pm 5^{\circ} \mathrm{C}$. Early devices which were placed on $200^{\circ} \mathrm{C}$ powered burn-in have shown only a few tenths of a degree $\mathrm{C}$ change in over $2000 \mathrm{~h}$ after a 100-h initial burn-in period.

\section{Application}

The current mode operation of this device lends itself to numerous applications. Fig. 8 is probably the simplest and most straightforward way to use the two-terminal temperature transducer as a centigrade thermometer. Resistor $R_{1}$ converts the current to an accurate voltage. Voltage divider $R_{2}$ and $R_{3}$ provides the Kelvin to centigrade conversion using whatever low TC reference is available. (In most cases, the

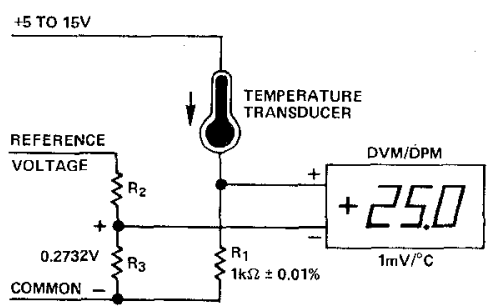

Fig. 8. Typical application as a centigrade thermometer.

reference and power supply are available from the meter.) For a Fahrenheit thermometer, the resistors are simply changed for a different scale factor and offset. For a Kelvin thermometer (or for centigrade with digital conversion from Kelvin), $R_{2}, R_{3}$, and the reference are not needed. The meter would then be connected directly across $R_{1}$. (The TC's of $R_{1}$ and the meter are, of course, critical in this circuit.) The current mode output also makes this device uniquely useful for creating a variety of linearly or functionally temperature dependent signals to use to compensate for known temperature coefficients.

\section{CONCLUSION}

The two-terminal IC temperature transducer described in this paper develops a total supply current that is directly proportional to absolute temperature by relying on a fundamental property of bipolar transistors. The inherent linearity, insensitivity to operating voltage, and laser trim calibration make the device uniquely useful and applicable among the other sensors presently available.

\section{REFERENCES}

[1] R. C. Dobkin, "Monolithic temperature transducer," in ISSCC Digest Tech. Papers, pp. 126-127, Feb. 1974.

[2] P. E. Gray et al., Physical Electronics and Circuit Models of Transistors. New York: Wiley, 1964.

[3] M. A. Maidique, "A high-precision monolithic super-beta operational amplifier," IEEE J. Solid-State Circuits, vol. SC-7, pp. 480 487, Dec. 1972.

[4] P. R. Holloway, "A high-yield, second generation 10-bit monolithic DAC," to be published in the IEEE J. Solid-State Circuits, Apr. 1977.

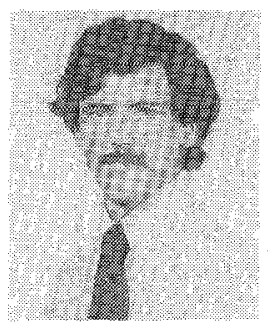

Michael P. Timko (M'76) was born in Ames, IA, on July 28,1947 . He received the B.S. and M.S. degrees from the Massachusetts Institute of Technology, Cambridge, in 1970.

Except for two intervening years in the U.S Air Force, he has worked for the Semiconductor Division of Analog Devices (formerly Nova Devices), Wilmington, MA, since 1970, where he is a Senior Design Engineer.

Mr. Timko is a member of Tau Beta Pi, Eta Kappa $\mathrm{Nu}$, and Sigma $\mathrm{Xi}$. 